\title{
Color magnetism in non-Abelian vortex matter
}

\author{
Michikazu Kobayashi, ${ }^{a}$ Eiji Nakano ${ }^{b}$ and Muneto Nitta $^{c}$ \\ ${ }^{a}$ Department of Physics, Kyoto University, \\ Oiwake-cho, Kitashirakawa, Sakyo-ku, Kyoto 606-8502, Japan \\ ${ }^{b}$ Department of Physics, Faculty of Science, Kochi University, \\ Kochi 780-8520, Japan \\ ${ }^{c}$ Department of Physics, and Research and Education Center for Natural Sciences, Keio University, \\ Hiyoshi 4-1-1, Yokohama, Kanagawa 223-8521, Japan \\ E-mail: michikaz@scphys.kyoto-u.ac.jp, e.nakano@kochi-u.ac.jp, \\ nitta@phys-h.keio.ac.jp
}

ABSTRACT: We propose color magnetism as a generalization of the ordinary Heisenberg (anti-)ferro magnets on a triangular lattice. Vortex matter consisting of an Abrikosov lattice of non-Abelian vortices with color magnetic fluxes shows a color ferro or anti-ferro magnetism, depending on the interaction among the vortex sites. A prime example is a nonAbelian vortex lattice in rotating dense quark matter, showing a color ferromagnetism. We show that the low-energy effective theory for the vortex lattice system in the color ferromagnetic phase is described by a $3+1$ dimensional $\mathbb{C} P^{N-1}$ nonlinear sigma model with spatially anisotropic couplings. We identify gapless excitations independent from Tkachenko modes as color magnons, that is, Nambu-Goldstone modes propagating in the vortex lattice with an anisotropic linear dispersion relation $\omega_{p}^{2}=c_{x y}^{2}\left(p_{x}^{2}+p_{y}^{2}\right)+c_{z}^{2} p_{z}^{2}$. We calculate the transition temperature between the ordered and disordered phases, and apply it to dense quark matter. We also identify the order parameter spaces for color anti-ferromagnets.

Keywords: Spontaneous Symmetry Breaking, Solitons Monopoles and Instantons, Sigma Models, Topological States of Matter

ARXIV EPRINT: 1311.2399 


\section{Contents}

1 Introduction 1

2 Non-Abelian vortex lattice 3

3 Color magnons in color ferromagnets 5

4 Color anti-ferromagnets $\quad 7$

$\begin{array}{lll}5 & \text { Summary and discussion } & 7\end{array}$

$\begin{array}{lll}\text { A Transition temperature within the mean-field analysis } & 8\end{array}$

$\begin{array}{ll}\text { B OPS of color anti-ferromagnets on a triangular lattice } & 10\end{array}$

\section{Introduction}

Magnetism is one of important subjects in condensed matter physics because of fundamental question of what the origin of magnets is and applications to the modern technology. The magnetism should be explained from the electromagnetic interaction. On the other hand, there are other fundamental forces in nature, such as the strong interaction acting on quarks and gluons with color degrees of freedom. Here, we report a new state of matter which we call "color magnetism," that may appear in non-Abelian vortex lattices when high density quark matter is rotating.

Quark matter at finite temperature and/or density is likely to have a rich phase structure due to a variety of internal symmetries [1]. At very high baryon number densities and low temperatures, quark matter is expected to exhibit a color superconductivity where condensation of quark Cooper pairs breaks color symmetry as well as flavor and the baryon number symmetries. For three colors and three massless flavors, the order parameter of the quark pair condensation is given by $[2,3]$

$$
\Phi_{a \alpha}=\epsilon_{a b c} \epsilon_{\alpha \beta \gamma}\left\langle\psi_{b \beta}^{T} C \gamma_{5} \psi_{c \gamma}\right\rangle=\Delta \delta_{a \alpha}
$$

where $a, b, c(\alpha, \beta, \gamma)$ represent the flavor (color) indices, and $\Delta$ is the quark pairing gap. The symmetry breaking pattern, $\mathrm{SU}(3)_{\mathrm{C}} \times \mathrm{SU}(3)_{\mathrm{L}} \times \mathrm{SU}(3)_{\mathrm{R}} \times \mathrm{U}(1)_{\mathrm{B}} \rightarrow \mathrm{SU}(3)_{\mathrm{C}+\mathrm{L}+\mathrm{R}}$, shows that this phase retains only a simultaneous rotation of color and flavors as the residual symmetry, thus is called color-flavor locking (CFL) phase. We denote the suffix $\mathrm{L}+\mathrm{R}$ by $\mathrm{F}$ in the following.

A possible candidate for system where the CFL phase takes place might be the core of very heavy compact stars, such as neuron stars. If the CFL phase develops in the rotating 
neutron star, vortices are created because of the superfluid nature associated with the $\mathrm{U}(1)_{\mathrm{B}}$ breaking in the CFL phase. The most fundamental vortex in the CFL phase is a non-Abelian vortex $[4,5]$, see ref. [6] as a review. The solution is of the form

$$
\Phi=\Delta \operatorname{diag}\left\{e^{i \theta} f(r), g(r), g(r)\right\}
$$

where $(\theta, r)$ are the polar coordinates for a vortex extended along the $z$ direction, and $f$ and $g$ are profile functions with suitable boundary conditions. Since the winding factor $e^{i \theta}$ is generated by both of the color $\mathrm{SU}(3)_{\mathrm{C}}$ and $\mathrm{U}(1)_{\mathrm{B}}$ rotations, the non-Abelian vortex carries a color-magnetic flux along the vortex core, and $1 / 3$ winding of the $\mathrm{U}(1)_{\mathrm{B}}$ phase as well. Therefore, the non-Abelian vortex possesses nature of both superconducting and superfluid vortices, associated with breakings of the $\mathrm{SU}(3)_{\mathrm{C}}$ color gauge symmetry and the baryon number $\mathrm{U}(1)_{\mathrm{B}}$ symmetry, respectively.

As the most remarkable property, the non-Abelian vortex has localized zero modes; the advent of the non-Abelian vortex further breaks the residual symmetry, $\mathrm{SU}(3)_{\mathrm{C}+\mathrm{F}} \rightarrow$ $[\mathrm{SU}(2) \times \mathrm{U}(1)]_{\mathrm{C}+\mathrm{F}}$, and thus there appear the associated Nambu-Goldstone (NG) modes which are identified as $\mathbb{C} P^{2}$ orientation modes [7, 8];

$$
\frac{\mathrm{SU}(3)_{\mathrm{C}+\mathrm{F}}}{[\mathrm{SU}(2) \times \mathrm{U}(1)]_{\mathrm{C}+\mathrm{F}}} \simeq \mathbb{C} P^{2}
$$

Since bulk side far from vortex core is approaching to the uniform CFL phase, the $\mathbb{C} P^{2}$ modes are normalizable, i.e., well localized along the vortex core. A different orientation of the $\mathbb{C} P^{2}$ modes corresponds to a different color of magnetic fluxes. Since the $\mathbb{C} P^{2}$ modes are transformed under $\mathrm{SU}(3)_{\mathrm{C}+\mathrm{F}}$ rotation as the fundamental representation, there are three eigenstates which we denote by a three-component complex variable $\phi$ as homogeneous coordinates of the $\mathbb{C} P^{2}$ space, where the $\phi$ satisfies a constraint $\phi^{\dagger} \phi=1$, and its overall phase factor is redundant by definition. Since the $\mathbb{C} P^{2}$ modes reside along the vortex line which we put along the $z$-axis, its low-energy effective theory on the vortex world sheet is obtained if the $\mathbb{C} P^{2}$ modes are promoted to $1+1$ dimensional fields $\phi(z, t)$ through the moduli space approximation [9]. It results in a nonlinear $\mathbb{C} P^{2}$ sigma model $[8,10]$,

$$
\mathcal{L}_{\mathbb{C} P^{2}}=\sum_{\mu=0,3} K_{\mu}\left[\partial^{\mu} \phi^{\dagger} \partial_{\mu} \phi+\left(\phi^{\dagger} \partial^{\mu} \phi\right)\left(\phi^{\dagger} \partial_{\mu} \phi\right)\right]
$$

where $K_{0,3}$ are called the Kähler class given by the integration in terms of the profile functions $f$ and $g$.

However, the above $\mathbb{C} P^{2}$ model in $1+1$ dimensions is gapped through quantum corrections $[11,12]$, because of the Coleman-Mermin-Wagner theorem [13, 14] forbidding the presence of NG modes or a long range order in $1+1$ dimensions.

In this Letter, we show that this is only the case with a single vortex. When there is a vortex lattice consisting of a huge number of vortices, as expected in rotating quark matter such as in the core of neutron stars, there exist $\mathbb{C} P^{2} \mathrm{NG}$ modes as gapless excitations in $3+1$ dimensions whose dispersion relation is linear and anisotropic. This is due to interactions between vortices depending on $\mathbb{C} P^{2}$ orientations [15]. We discuss transition temperature 
between ordered and disordered phases and find that non-Abelian vortex matter form a color ferromagnet.

Apart from dense quark matter, non-Abelian vortices with $\mathbb{C} P^{N-1}$ modes were found in supersymmetric $\mathrm{U}(N)$ gauge theories with $N$ flavors $[16,17]$ (see $[18-20]$ as a review). The low-energy effective theory of a vortex is the $\mathbb{C} P^{N-1}$ model which is in the same form with eq. (1.4) if we interpret $\phi$ as a complex $N$ vector. Supersymmetric theories usually admit Bogomol'nyi-Prasado-Sommerfield (BPS) vortices among which no static interaction exist. In this case, multiple vortices stably exist at arbitrary separations [21, 22]. On the other hand, here we are interested in non-BPS vortices among which static force exist [15]. In the following, we consider general $N$.

\section{Non-Abelian vortex lattice}

We discuss a color ferromagnetism of $\mathbb{C} P^{N-1}$ on a vortex lattice system. In rotating systems of CFL quark matter, multiple non-Abelian vortices are created and may form a vortex lattice. In general, structure of the vortex lattice is determined by vortex-vortex interaction. In the present case of non-Abelian vortices, the interaction is mediated by massless $\mathrm{U}(1)_{\mathrm{B}}$ phonons [7, 23], and also by scalars and gluons [24] which are massive, where the scalars are amplitude fluctuations of the gap function $\Phi$. If the vortex lattice is dilute, i.e., the inter vortex distance is large, the vortex-vortex interaction is dominated by the $\mathrm{U}(1)_{\mathrm{B}}$ phonons which provide a long-range repulsion in the same manner of usual superfluid vortices. Thus the structure of the dilute vortex lattice must be the Abrikosov's triangular lattice [7, 23].

We first define the system of our interest more precisely: vortex lattice has the triangle structure of spacing $L$ in the $x-y$ plane, and these vortices are set parallel to each other along the $z$ direction. Once locations of vortices are fixed on the triangular lattice sites by the repulsive force, relative orientation of the $\mathbb{C} P^{N-1}$ modes between neighboring vortices are determined solely by the interaction mediated by massive scalars and gluons. The $\mathbb{C} P^{N-1}$ dependent vortex-vortex interaction has already been derived in the previous study [6], and $V_{\text {int }}^{\langle i, j\rangle}$, the interaction energy per unit length in the $z$-direction, is of the form

$$
V_{\mathrm{int}}^{\langle i, j\rangle} / \Delta^{2}=\sum_{A} G_{\langle i, j\rangle, A}\left(\phi_{i}^{\dagger} T_{A} \phi_{i}\right)\left(\phi_{j}^{\dagger} T_{A} \phi_{j}\right)
$$

where $\langle i, j\rangle$ represents sites of two adjacent vortices, $G_{\langle i, j\rangle, A}$ is the interaction strength between them, and $T_{A}, A=1, \cdots, N^{2}-1$, are generators of $\mathrm{SU}(N)$. The above expression is the leading order result under assumption that well separated two vortices are located parallel and their relative $\mathbb{C} P^{N-1}$ orientations are constant along vortex line. $G_{\langle i, j\rangle, A}$ includes contributions from gluon-exchange and scalar-exchange potentials. The $\mathrm{U}(1)_{\mathrm{B}}$ phonon does not feel the $\mathbb{C} P^{N-1}$ orientation, thus gives no contribution. In the context of quark matter, the gluon exchange part of the above $\mathbb{C} P^{N-1}$ dependent interaction was devised [6] from the dual transformation which was used [24] to describe a topological interaction between non-Abelian vortex and quasiparticles such as gluons and phonon in the bulk space, while, at present, we do not have a such systematic derivation for coupling between the scalar fields and non-Abelian vortex. Nevertheless, a seminal work [15] on 
numerical simulation on the potential energy between two non-BPS non-Abelian vortices provides implication of an asymptotic form of full $G_{\langle i, j\rangle, A}$ including both contributions. With inter vortex distance $r$ being defined and isotropy in the color space $A$, the asymptotic form of $G(r)=G_{\langle i, j\rangle, A}$ would be given by [15]

$$
G(r) \simeq-C_{1} B_{0}\left(m_{\chi} r\right)+C_{2} B_{0}\left(m_{g} r\right)
$$

where $C_{1}$ and $C_{2}$ are positive numerical factors, and $B_{0}(r)$ the modified Bessel function, $B_{0}(m r) \sim \sqrt{\pi / 2 m r} e^{-m r}$ for $m r \gg 1$. In the above equation, the first term corresponds to massive scalar contributions and the second to massive gluon contributions, and it should be noted that the massive gluons and scalars give the opposite sign of interaction. For sufficiently large lattice spacing $L$, contribution of the lightest particle dominates the interaction.

The $\mathbb{C} P^{N-1}$ modes live in each vortex site, and their dynamics is described by the following effective Hamiltonian,

$$
\begin{aligned}
H & =\int d z \sum_{\langle i, j\rangle, A}\left[-J_{x y} S_{i, A} S_{j, A}+K_{3}\left\{\left|\partial_{z} \phi_{i}\right|^{2}+\left(\phi_{i}^{\dagger} \partial_{z} \phi_{i}\right)^{2}\right\}\right] \\
S_{i, A} & :=\phi_{i}^{\dagger} T_{A} \phi_{i}, \quad J_{x y}:=\Delta^{2} G(L)
\end{aligned}
$$

where $S_{i, A}$ are "spin" variables and $J_{x y}$ is the interaction between neighboring vortices.

If $\phi$ is uniform in the $z$-direction, this Hamiltonian reduces to the Heisenberg model for $N=2$. In this case, $J_{x y}>0$ and $J_{x y}<0$ correspond to ferromagnets and antiferromagnets, respectively. We use these terminologies for general $N$.

The model with $N \geq 3$ further provides higher-dimensional nontrivial spin system as a new statistical model. The case of $N=3$ corresponds to color magnetism in rotating dense quark matter discussed here. First, the Kähler class $K_{0,3}$ is estimated as $K_{0} \simeq$ $3 K_{3} \simeq 3 \mu^{2} / \Delta^{2}$ at very high density [10]. The interaction $J_{x y}$ is determined through the masses of particles which vortices exchange. At very high densities, the masses are given as $m_{s}=2 m_{\chi} \sim 2 \Delta, m_{g} \sim g \mu$, where $m_{s, \chi}$ corresponds to the singlet (adjoint) part of scalar fields in the residual $\mathrm{SU}(3)_{\mathrm{C}+\mathrm{F}}$ symmetry, $\mu$ is the baryon number chemical potential, and $g$ is coupling constant of strong interaction. At very high densities, i.e., very large $\mu, g$ and $\Delta$ are exponentially small with respect to $\mu$, therefore, one expects $m_{g} \gg m_{s}, m_{\chi}$. Thus,

$$
G(L) \simeq-C_{1} \sqrt{\frac{\pi}{2 m_{\chi} L}} e^{-m_{\chi} L}
$$

Therefore, in the case of rotating CFL quark matter, because of the sign of the interaction above, the nearest neighbor interaction of $\mathbb{C} P^{N-1}$ modes is found to align their orientations (color fluxes), leading to a color ferromagnetism.

An estimation of $J_{x y}$ is given as follows. Supposing the present system is inside of a neutron star of the radius $R$ with rotation period $P_{\text {rot }}$, we can valuate a ballpark of the number of vortex as $N_{v} \simeq 1.9 \times 10^{19}\left(\frac{1 \mathrm{~ms}}{P_{\mathrm{rot}}}\right)\left(\frac{\mu / 3}{300 \mathrm{MeV}}\right)\left(\frac{R}{10 \mathrm{~km}}\right)$, from which the lattice spacing is estimated [25] as $L \equiv\left(\frac{\pi R^{2}}{N_{v}}\right)^{1 / 2} \simeq 4.0 \times 10^{-6} \mathrm{~m}\left(\frac{P_{\mathrm{rot}}}{1 \mathrm{~ms}}\right)^{1 / 2}\left(\frac{300 \mathrm{MeV}}{\mu / 3}\right)^{1 / 2}$. A set of values 
of $C_{1} \sim 10, L \simeq 4.0 \times 10^{-6} \mathrm{~m}$, and the gap energy $\Delta \sim 100 \mathrm{MeV}$ for low temperatures $[2,26]$ gives $\log \left(J_{x y} / \Delta^{2}\right) \sim-10^{9}$, which is quite small in comparison to the coupling in the $z$ direction. To have $J_{x y} / \Delta^{2}=O(1)$, we need to put the system very close to the critical point $\left(T_{\mathrm{c}}^{\mathrm{CFL}}-T\right) / T_{\mathrm{c}}^{\mathrm{CFL}} \sim 10^{-18}$, where we have used a mean-field result $\Delta \simeq 2.16 T_{\mathrm{c}}^{\mathrm{CFL}} \sqrt{1-T / T_{\mathrm{c}}^{\mathrm{CFL}}}[27]$, or we have to set an asymptotically high density.

Here let us point out that the smallness of the effective coupling $J_{x y}$ in the above estimation is based upon multiple approximations, i.e., the moduli space approximation and an asymptotic form of the interaction (2.2), both of which work against correlations among orientions in the $x-y$ plane. What we claim here is that the present study provides the qualitative argument on how orientational modes make order of color magnetism, which is common feature for general non-BPS vortex lattice systems [15]. So actual correlations amog orientations in the $x-y$ plane are more measurable, at least, for higher vortex densities.

\section{Color magnons in color ferromagnets}

Low-energy excitations in the ordered phase have an important role in determination of, e.g., thermodynamic and transport properties of the system. The present system exhibits a color ferromagnetism, thus we expect that there appear massless excitations like magnons in the ordinary ferromagnetism, which correspond to fluctuations of the $\mathbb{C} P^{N-1}$ modes around an orientation spontaneously set in color ferromagnetic phase. To investigate dynamics of these excitations, we take a long wavelength limit of the interaction between neighboring $\mathbb{C} P^{N-1}$ modes, eq. (2.1), which can be described by a continuum limit of the interaction. By use of Fierz transformation, $\sum_{A}\left(T_{A}\right)_{b}^{a}\left(T_{A}\right)_{d}^{c}=\delta_{d}^{a} \delta_{b}^{c} / 2-\delta_{b}^{a} \delta_{d}^{c} / 2 N(a, b, c, d=1, \cdots, N)$, we can take a continuum limit of each bond of the triangle lattice in eq. (2.3), up to irrelevant constants,

$$
\sum_{A}\left(\phi_{i}^{\dagger} T_{A} \phi_{i}\right)\left(\phi_{j}^{\dagger} T_{A} \phi_{j}\right) \rightarrow-\frac{L^{2}}{2}\left[|\nabla \phi|^{2}+\left(\phi^{\dagger} \nabla \phi\right)^{2}+\mathcal{O}\left(\nabla^{4}\right)\right],
$$

for a pair of the nearest neighbors $i, j$. Then, we sum all bonds to obtain

$$
-\sum_{\langle i, j\rangle, A} J_{x y}\left(\phi_{i}^{\dagger} T_{A} \phi_{i}\right)\left(\phi_{j}^{\dagger} T_{A} \phi_{j}\right) \rightarrow \tilde{K}_{x y} \int \mathrm{d} x \mathrm{~d} y \sum_{i=x, y}\left[\left|\partial_{i} \phi\right|^{2}+\left(\phi^{\dagger} \partial_{i} \phi\right)^{2}\right],
$$

with a coupling constant being defined by $\tilde{K}_{x y}=J_{x y}\left(3 L^{2} / 2\right)\left(2 / L^{2} \sqrt{3}\right)(1 / 2)=\sqrt{3} J_{x y} / 2$.

Incorporating the effective theory in the $z$ and temporal directions with redefined couplings $\tilde{K}_{0,3}=2 K_{0,3} / L^{2} \sqrt{3}$, where $2 / L^{2} \sqrt{3}$ is the vortex density in the $x$-y plane, we obtain a low-energy effective theory for the magnon modes in $3+1$ dimension, $L_{\text {eff }}=$ $\int \mathrm{d} x \mathrm{~d} y \mathrm{~d} z \mathcal{L}_{\text {eff }}$ with

$$
\mathcal{L}_{\text {eff }}=\sum_{\mu=0}^{3} \tilde{K}_{\mu}\left[\partial^{\mu} \phi^{\dagger} \partial_{\mu} \phi+\left(\phi^{\dagger} \partial^{\mu} \phi\right)\left(\phi^{\dagger} \partial_{\mu} \phi\right)\right]
$$

which is a $\mathbb{C} P^{N-1}$ model with spatially anisotropic couplings; $\tilde{K}_{1}=\tilde{K}_{2}=\tilde{K}_{x y} \neq \tilde{K}_{3}$. 
Now let us study the low-energy excitations of this model. To this end, we can set an orientation of the ground state as $\phi_{0}=(1,0, \cdots, 0)$ without loss of generality. The color magnon corresponds to fluctuations around the orientation, $\phi=U\left(\delta_{a}\right) \phi_{0}=\phi_{0}+\tilde{\phi}+O\left(\tilde{\phi}^{2}\right)$, where $U\left(\delta_{a}\right) \in \mathrm{SU}(N)_{\mathrm{C}+\mathrm{F}}$ and $\tilde{\phi}:=i X_{a} \delta_{a} \phi_{0}=\left(i \delta_{0},-\delta_{1}+i \delta_{2}, \cdots,-\delta_{2 N-3}+i \delta_{2(N-1)}\right)^{T}$. Parameters $\delta_{a}$ are assigned to broken generators $X_{a}$ of the dimension of $\mathrm{SU}(N) / \mathrm{SU}(N-1)$, and are identified as the color magnons. The fluctuation $\delta_{0}$ is eliminated by overall $\mathrm{U}(1)$ operation, thus will be redundant. Plugging these expression into the effective Lagrangian, we have dynamics of the color magnons as

$$
L_{\text {eff }}=\sum_{\mu=0}^{3} \tilde{K}_{\mu} \operatorname{Tr}\left[\left(1-\phi_{0} \phi_{0}^{\dagger}\right) \partial^{\mu} \tilde{\phi} \partial_{\mu} \tilde{\phi}^{\dagger}\right]+O\left(\tilde{\phi}^{3}\right)=\sum_{\mu=0}^{3} \sum_{a=1}^{2(N-1)} \tilde{K}_{\mu} \partial^{\mu} \delta_{a} \partial_{\mu} \delta_{a}+O\left(\delta_{a}^{3}\right) .
$$

Then, the dispersion relation can be obtained as

$$
\omega_{p}^{2}=\left(\tilde{K}_{x y}\left(p_{x}^{2}+p_{y}^{2}\right)+\tilde{K}_{z} p_{z}^{2}\right) / \tilde{K}_{0},
$$

which shows that the color magnons propagate in the $x, y$ directions with velocity $c_{x y}^{2}=$ $\tilde{K}_{x y} / \tilde{K}_{0}$ and the $z$-direction with $c_{z}^{2}=\tilde{K}_{3} / \tilde{K}_{0}$. In the case of $N=3$ for the CFL quark matter, $c_{z}^{2}=K_{3} / K_{0} \simeq 1 / 3$, and $c_{x y}^{2}=\Delta^{4} G(L) L^{2} \sqrt{3} / 2 \mu^{2} \simeq a_{2} t^{7 / 4} e^{-a_{1} \sqrt{t}}$ where $t=$ $\left(T_{\mathrm{c}}^{\mathrm{CFL}}-T\right) / T_{\mathrm{c}}^{\mathrm{CFL}}$, which is obtained from a mean-field result at very high densities. This formula gives the maximal value of $c_{x y}$ with respect to temperature, which is achieved at $t=\left(7 / 2 a_{1}\right)^{2}$. For typical values of parameters, $a_{1} \sim 10^{9}$, and $a_{2} \sim 10^{12.5}, c_{x y} \sim 10^{-9}$ (= $0.3 \mathrm{~m} / \mathrm{s}$ ) at most, giving 9 hours oscillation for a neutron star with $R=10 \mathrm{~km}$.

The color magnons are massless excitations propagating with a linear dispersion (of type I) independently from the other NG modes, e.g., Kelvin/Tkachenko modes associated with the breaking of translational invariance, which have a quadratic dispersion (of type II).

For a single non-Abelian vortex system, where the effective theory is given by the $1+1$ dimensional $\mathbb{C} P^{N-1}$ model, there is no ordered phase because of strong quantum fluctuation effects. For the present vortex lattice system, however, an ordered phase can be expected because the fluctuations are suppressed by propagating along the $x y$-directions in the longwavelength limit, and the mean-field analysis becomes better in such a $3+1$ dimensional system compared to lower dimensional systems. Within the mean-field analysis, the critical temperature $T_{\mathrm{c}}^{\mathrm{order}}$, below which the vortex lattice system is ordered, can be estimated as (see appendix A for derivation)

$$
T_{\mathrm{c}}^{\text {order }} \sim \frac{J_{x y}}{k_{\max }}+K_{3} k_{\max }
$$

where the first and second terms correspond to the mean-field critical temperature along the $x y$ and $z$-directions, respectively, and $k_{\max }$ is the maximum wavenumber taken into account along the $z$-direction. In the continuum limit $k_{\max } \rightarrow \infty$ for the $z$-direction, the transition temperature diverges, which implies the ordered phase and the existence of the corresponding color magnon excitations in the whole temperature region. However, the divergent temperature is a mean-field artifact, and the disordered phase may be expected at the finite temperature within the effect beyond mean-field analysis. 


\section{Color anti-ferromagnets}

Although the color magnetism with $J_{x y}>0$ is only the physical system with $N \geq 3$ known thus far, it may be worth to mention basic properties of color anti-ferromagnets with $J_{x y}<0$.

We denote the order parameter space (OPS) for anti-ferromagnets by $\mathcal{M}_{\mathrm{AF}}^{N=2}$. We then find (see appendix B for derivation)

$$
\begin{aligned}
& \mathcal{M}_{\mathrm{AF}}^{N=2} \simeq \mathrm{SU}(2) / \mathbb{Z}_{2} \simeq \mathrm{SO}(3) \\
& \mathcal{M}_{\mathrm{AF}}^{N=3} \simeq \mathrm{SU}(3) / \mathrm{U}(1)^{2}
\end{aligned}
$$

The OPS for $N=2$, $\mathrm{SO}(3)$, is well-known for Heisenberg anti-ferromagnets [28, 29] while the second is a so-called flag manifold of rank two. The first and second homotopy groups of the OPS are important for the presence or absence of a Kosterlitz-Thouless (KT) phase transition [28]. They are

$$
\begin{aligned}
& \pi_{1}\left(\mathcal{M}_{\mathrm{AF}}^{N=2}\right) \simeq \mathbb{Z}_{2}, \\
& \pi_{2}\left(\mathcal{M}_{\mathrm{AF}}^{N=2}\right) \simeq 0 \\
& \pi_{1}\left(\mathcal{M}_{\mathrm{AF}}^{N=3}\right) \simeq 0, \\
& \pi_{2}\left(\mathcal{M}_{\mathrm{AF}}^{N=3}\right) \simeq \mathbb{Z} \oplus \mathbb{Z} \text {. }
\end{aligned}
$$

The first homotopy groups show that the KT transition can happen only for $N=2$. The second homotopy groups imply the existence of two-dimensional Skyrmions (lumps) extended as strings for $N=3$.

\section{Summary and discussion}

As a generalization of the ordinary magnetism, we have found color magnets in lattices consisting of non-Abelian vortices with $\mathbb{C} P^{N-1}$ internal orientations. While the case of $N=2$ reduces to Heisenberg (anti-)ferromagnets, rotating dense quark matter provides an example of color ferromagnets in the $N=3$ case. $^{1}$

For color ferromagnets, we have found color magnons as gapless NG modes propagating in $3+1$ dimensions with the dispersion relation anisotropic in the direction along the rotating axis and the orthogonal plane, and have estimated the transition temperature in the mean-field approximation. For color anti-ferromagnets, we have found the OPS and relevant homotopy groups.

When we take into account the electromagnetic interactions $\mathrm{U}(1)_{\mathrm{EM}}$, the $\mathbb{C} P^{2}$ modes of a non-Abelian vortex are electrically charged and the interaction between a vortex and the electromagnetic field is described by a $\mathrm{U}(1)_{\mathrm{EM}}$ gauged $\mathbb{C} P^{2}$ model [25]. Hence, the $\mathbb{C} P^{2}$ modes affect the electric conductivity in addition to the thermal conductivity. There should be anisotropy on these conductivities, which may affect evolution of neutron stars such as the cooling process of the star, etc. As for electric conductivity, it was predicted

\footnotetext{
${ }^{1}$ In response to an external magnetic field other vortex solution can be found, where gluons are condensed due to a chromomagnetic instability [30,31]. Since the non-Abelian vortex we have discussed here generates a sponteneous magnetic field if the electromagnetism is introduced [32], investigation of their interplay is of interest as a future work.
} 
that electro-magnetic waves along a vortex lattice decay and therefore the CFL phase acts as a polarizer [25].

Another effect of the electromagnetic interactions is a mixing between $\mathrm{U}(1)_{\mathrm{EM}}$ and the $\lambda_{8}$ component of strong interaction. This induces a tension difference among the $\mathbb{C} P^{2}$ degenerated vortices [32]. This effect can be incorporated by an effective potential in the $\mathbb{C} P^{2}$ model.

A lattice formulation of the $\mathbb{C} P^{N-1}$ model was proposed by using a $\mathrm{U}(1)$ gauge field on link variables $[33,34]$. Our model in eq. (3.1) provides a simpler lattice formulation without gauge fields.

\section{Acknowledgments}

This work is supported in part by Grant-in-Aid for Scientific Research (Grant No. 22740219 (M.K.),No. 24740166 (E.N.), and No. 25400268 (M.N.)) and the work of M.N. is also supported in part by the "Topological Quantum Phenomena" Grant-in-Aid for Scientific Research on Innovative Areas (No. 25103720) from the Ministry of Education, Culture, Sports, Science and Technology (MEXT) of Japan.

\section{A Transition temperature within the mean-field analysis}

We start from the system in the discrete layered triangular lattice:

$$
H=-\sum_{i \in(x, y, z), A}\left(J_{x y}^{\mathrm{dis}} \sum_{j \in N N_{i}(x, y)} S_{i A} S_{j A}+J_{z}^{\mathrm{dis}} \sum_{k \in N N_{i}(z)} S_{i A} S_{k A}\right)
$$

where $S_{i A} \equiv \phi_{i}^{\dagger} T_{A} \phi_{i}, N N_{i}(x, y)$ is the nearest sites to $i$ th site for the triangular lattice in the $x y$-plane, and $N N_{i}(z)$ is the site in the nearest layer perpendicular to the $z$-axis. Dividing $S_{i A}$ into the mean field and its fluctuation, i.e., $S_{i A}=\left\langle S_{A}\right\rangle+\delta S_{i A}$ and neglecting the second ordered fluctuation $\delta S_{i A} \delta S_{j A}$, we obtain

$$
\begin{aligned}
H & =\tilde{J} \sum_{A}\left(\frac{N_{x y} N_{z}\left\langle S_{A}\right\rangle^{2}}{2}-\sum_{i}\left\langle S_{A}\right\rangle S_{i A}\right) \\
\tilde{J} & \equiv\left(6 J_{x y}^{\mathrm{dis}}+2 J_{z}^{\mathrm{dis}}\right),
\end{aligned}
$$

where 6 and 2 in $\tilde{J}$ indicate the number of nearest sites for the triangular lattice in the $x y$-plane and the layers perpendicular to the $z$-axis, and $N_{x y}$ and $N_{z}$ are the total number of the triangular lattice sites and layers respectively. The partition function $Z$ written as

$$
\begin{aligned}
Z & =e^{-\beta E_{0}} \int \prod_{i}\left(\prod_{A} D \psi_{i A}\right) \times \delta\left(\sum_{A}\left|\psi_{i A}\right|^{2}-1\right) e^{\beta \tilde{J} \sum_{A}\left\langle S_{A}\right\rangle S_{i A}} \equiv e^{-\beta E_{0}} \prod_{i} Z_{i} \\
E_{0} & =E_{0}\left(\left\langle S_{A}\right\rangle\right) \equiv \frac{N_{x y} N_{z} \tilde{J}}{2} \sum_{A}\left\langle S_{A}\right\rangle^{2}
\end{aligned}
$$


can be calculated by diagonalizing $\sum_{A}\left\langle S_{A}\right\rangle S_{i A}$. For $\mathbb{C} P^{N-1}$ case, $Z_{i}$ is formally written as $Z_{i} \equiv Z_{N}\left(B_{1}, \cdots, B_{N}\right)$ where, $B_{i}$ is the $i$ th eigenvalue for $\sum_{A}\left\langle S_{A}\right\rangle S_{i A}$ satisfying $\sum_{i} B_{i}=0$. $Z_{N}$ satisfies the following recurrence relation:

$$
\begin{aligned}
\beta \tilde{J} Z_{N}\left(B_{1}, \cdots, B_{N}\right) & =\frac{\pi}{B_{N-1}-B_{N}}\left\{Z_{N-1}\left(B_{1}, B_{N-2}, B_{N-1}\right)-Z_{N-1}\left(B_{1}, B_{N-2}, B_{N}\right)\right\}, \\
\beta \tilde{J} Z_{2}\left(B_{1}, B_{2}\right) & =\frac{2 \pi^{2}\left(e^{B_{1}}-e^{B_{2}}\right)}{B_{1}-B_{2}} .
\end{aligned}
$$

For $N=3$, eigenvalues $B_{1}, B_{2}, B_{3}=1-B_{1}-B_{2}$ further satisfy $B_{1}^{2}+B_{2}^{2}+B_{3}^{2}=$ $2 \beta \tilde{J} \sum_{A}\left\langle S_{A}\right\rangle^{2}$. The free energy $F$ becomes

$$
\begin{aligned}
\frac{F}{N_{x y} N_{z} \tilde{J}}= & \frac{B_{1}^{2}+B_{2}^{2}+B_{1} B_{2}}{2} \\
& -\frac{1}{\beta \tilde{J}}\left[\log \left\{\left(B_{1}+2 B_{2}\right) e^{\beta \tilde{J} B_{1}}+\left(B_{1}-B_{2}\right) e^{-\beta \tilde{J}\left(B_{1}+B_{2}\right)}-\left(2 B_{1}+B_{2}\right) e^{\beta \tilde{J} B_{2}}\right\}\right. \\
& \left.\quad-\log \left\{\left(B_{1}-B_{2}\right)\left(B_{1}+2 B_{2}\right)\left(2 B_{1}+B_{2}\right)\right\}+\log \left(2 \pi^{3}\right)-2 \log (\beta \tilde{J})\right] \\
\geqq & \frac{3 B_{1}^{2}}{2}-\frac{1}{\beta \tilde{J}}\left[\log \left\{1+e^{3 \beta \tilde{J} B_{1}}\left(3 \beta \tilde{J} B_{1}-1\right)\right\}\right. \\
\quad & \left.\quad-2 \beta \tilde{J} B_{1}-2 \log \left(3 B_{1}\right)+\log \left(2 \pi^{3}\right)-2 \log (\beta \tilde{J})\right] \\
= & -\frac{3 \log (\pi)}{\beta \tilde{J}}+\frac{1}{4}(6-\beta \tilde{J}) B_{1}^{2}+\frac{\beta^{2} \tilde{J}^{2}}{30} B_{1}^{3}+\frac{\beta^{3} \tilde{J}^{3}}{160} B_{1}^{4}+O\left(B_{1}^{5}\right) .
\end{aligned}
$$

In the second line, the equality is hold for $B_{2}=B_{1}, 2 B_{1}$, or $B_{1} / 2$. With this free energy, the first ordered phase transition occurs at $T_{\mathrm{c}}^{\text {order }} \simeq 53 \tilde{J} / 270=53\left(3 J_{x y}^{\text {dis }}+J_{z}^{\text {dis }}\right) / 135$.

Next, we consider the continuum limit of the Hamiltonian (A.1). Defining the distance between two layers as $\Delta_{z}=1 / k_{\max }$ and writing the continuous form as $\sum_{i \in z} \Delta_{z} \rightarrow \int d z$, we obtain the Hamiltonian (A.1)

$$
\begin{aligned}
H= & \frac{1}{\Delta_{z}} \sum_{i \in(x, y)} \int d z\left[-J_{x y}^{\mathrm{dis}} \sum_{j \in N N_{i}(x, y), A} S_{i A} S_{j A}+2 J_{z}^{\mathrm{dis}}\left\{\Delta_{z}^{2}\left|\partial_{z} \psi_{i}\right|^{2}+\Delta_{z}^{2}\left(\psi_{i}^{\dagger} \partial_{z} \psi_{i}\right)^{2}+O\left(\Delta_{z}^{4}\right)\right\}\right] \\
& -4 J_{z}^{\mathrm{dis}} N_{x y} N_{z} .
\end{aligned}
$$

Compared to the original effective Hamiltonian (2.3), we get the coupling constants

$$
\begin{aligned}
& J_{x y}^{\mathrm{dis}}=J_{x y} \Delta_{z}=\frac{J_{x y}}{k_{\max }}, \\
& J_{z}^{\text {dis }}=\frac{K_{3}}{2 \Delta_{z}}=\frac{K_{3} k_{\max }}{2},
\end{aligned}
$$

and the critical temperature

$$
T_{\mathrm{c}}^{\text {order }}=\frac{159 J_{x y}}{135 k_{\max }}+\frac{53 K_{3} k_{\max }}{270}
$$

which diverges in the continuous limit $k_{\max } \rightarrow \infty$. 


\section{B OPS of color anti-ferromagnets on a triangular lattice}

Here we derive the OPS for color anti-ferromagnets on a triangular lattice by generalizing that of Heisenberg anti-ferromagnets.

First, we recall the case of $N=2$, Heisenberg anti-ferromagnets, in which the target space is $\mathbb{C} P^{1} \simeq S^{2}$. With picking up one point on a triangular lattice, we fix a point on $S^{2}$. We then look at two nearest points of a triangle. The degrees of freedom to chose two points with an angle $2 \pi / 3$ with the first point of the $S^{2}$ is $S^{1} / \mathbb{Z}_{2}$. Then the OPS is $S^{1} / \mathbb{Z}_{2}$ fibered over $S^{2}$ :

$$
\mathcal{M}_{\mathrm{AF}}^{N=2} \simeq S^{1} / \mathbb{Z}_{2} \ltimes S^{2} \simeq S^{3} / \mathbb{Z}_{2} \simeq \mathrm{SO}(3) .
$$

The triangular lattice is filled by this set of triangle.

For higher $N \geq 3$, it is convenient to use homogeneous coordinates $\phi$ (a complex $N$-vector) of $\mathbb{C} P^{N-1}$. We have a constraint $\phi^{\dagger} \phi=1$ and the overall phase is redundant.

Let us consider the case of $N=3, \mathbb{C} P^{2} \simeq \mathrm{SU}(3) /[\mathrm{SU}(2) \times \mathrm{U}(1)]$. We take $\phi_{1}$ at one point of a triangular lattice to be $\phi_{1}^{T}=(1,0,0)$ without loss of generality. Next, we take $\phi_{2}^{T}=(0,1,0) \phi_{3}^{T}=(0,0,1)$ without loss of generality. Since the overall phase is redundant in each site, the symmetry $\mathrm{SU}(3)$ is broken to $\mathrm{U}(1)^{2}$. We then conclude

$$
\mathcal{M}_{\mathrm{AF}}^{N=3} \simeq \mathrm{SU}(3) / \mathrm{U}(1)^{2} .
$$

Open Access. This article is distributed under the terms of the Creative Commons Attribution License (CC-BY 4.0), which permits any use, distribution and reproduction in any medium, provided the original author(s) and source are credited.

\section{References}

[1] K. Fukushima and T. Hatsuda, The phase diagram of dense QCD, Rept. Prog. Phys. 74 (2011) 014001 [arXiv: 1005.4814] [INSPIRE].

[2] M.G. Alford, K. Rajagopal and F. Wilczek, Color flavor locking and chiral symmetry breaking in high density QCD, Nucl. Phys. B 537 (1999) 443 [hep-ph/9804403] [INSPIRE].

[3] M.G. Alford, A. Schmitt, K. Rajagopal and T. Schäfer, Color superconductivity in dense quark matter, Rev. Mod. Phys. 80 (2008) 1455 [arXiv: 0709.4635] [INSPIRE].

[4] A.P. Balachandran, S. Digal and T. Matsuura, Semi-superfluid strings in high density QCD, Phys. Rev. D 73 (2006) 074009 [hep-ph/0509276] [INSPIRE].

[5] M. Eto and M. Nitta, Color magnetic flux tubes in dense QCD, Phys. Rev. D 80 (2009) 125007 [arXiv:0907.1278] [InSPIRE].

[6] M. Eto, Y. Hirono, M. Nitta and S. Yasui, Vortices and other topological solitons in dense quark matter, Prog. Theor. Exp. Phys. 2014 (2013) 012D01 [arXiv:1308.1535] [InSPIRE].

[7] E. Nakano, M. Nitta and T. Matsuura, Non-Abelian strings in high density QCD: zero modes and interactions, Phys. Rev. D 78 (2008) 045002 [arXiv:0708.4096] [INSPIRE].

[8] M. Eto, E. Nakano and M. Nitta, Effective world-sheet theory of color magnetic flux tubes in dense QCD, Phys. Rev. D 80 (2009) 125011 [arXiv:0908.4470] [INSPIRE]. 
[9] N.S. Manton, A remark on the scattering of BPS monopoles, Phys. Lett. B 110 (1982) 54 [INSPIRE].

[10] M. Eto, M. Nitta and N. Yamamoto, Instabilities of non-Abelian vortices in dense QCD, Phys. Rev. Lett. 104 (2010) 161601 [arXiv:0912.1352] [INSPIRE].

[11] A. Gorsky, M. Shifman and A. Yung, Confined magnetic monopoles in dense QCD, Phys. Rev. D 83 (2011) 085027 [arXiv:1101.1120] [InSPIRE].

[12] M. Eto, M. Nitta and N. Yamamoto, Confined monopoles induced by quantum effects in dense QCD, Phys. Rev. D 83 (2011) 085005 [arXiv:1101.2574] [INSPIRE].

[13] S.R. Coleman, There are no Goldstone bosons in two-dimensions, Commun. Math. Phys. 31 (1973) 259 [InSPIRE].

[14] N.D. Mermin and H. Wagner, Absence of ferromagnetism or antiferromagnetism in one-dimensional or two-dimensional isotropic Heisenberg models, Phys. Rev. Lett. 17 (1966) 1133 [inSPIRE].

[15] R. Auzzi, M. Eto and W. Vinci, Static interactions of non-Abelian vortices, JHEP 02 (2008) 100 [arXiv:0711.0116] [INSPIRE].

[16] A. Hanany and D. Tong, Vortices, instantons and branes, JHEP 07 (2003) 037 [hep-th/0306150] [INSPIRE].

[17] R. Auzzi, S. Bolognesi, J. Evslin, K. Konishi and A. Yung, Non-Abelian superconductors: vortices and confinement in $N=2$ SQCD, Nucl. Phys. B 673 (2003) 187 [hep-th/0307287] [INSPIRE].

[18] M. Eto, Y. Isozumi, M. Nitta, K. Ohashi and N. Sakai, Solitons in the Higgs phase: the moduli matrix approach, J. Phys. A 39 (2006) R315 [hep-th/0602170] [INSPIRE].

[19] M. Shifman and A. Yung, Supersymmetric solitons and how they help us understand non-Abelian gauge theories, Rev. Mod. Phys. 79 (2007) 1139 [hep-th/0703267] [INSPIRE].

[20] M. Shifman and A. Yung, Supersymmetric solitons, Cambridge Monographs on Mathematical Physics, Cambridge University Press, Cambridge U.K. (2009) [INSPIRE].

[21] M. Eto, Y. Isozumi, M. Nitta, K. Ohashi and N. Sakai, Moduli space of non-Abelian vortices, Phys. Rev. Lett. 96 (2006) 161601 [hep-th/0511088] [INSPIRE].

[22] M. Eto et al., Non-Abelian vortices of higher winding numbers, Phys. Rev. D 74 (2006) 065021 [hep-th/0607070] [INSPIRE].

[23] E. Nakano, M. Nitta and T. Matsuura, Non-Abelian strings in hot or dense QCD, Prog. Theor. Phys. Suppl. 174 (2008) 254 [arXiv:0805.4539] [INSPIRE].

[24] Y. Hirono, T. Kanazawa and M. Nitta, Topological interactions of non-Abelian vortices with quasi-particles in high density QCD, Phys. Rev. D 83 (2011) 085018 [arXiv:1012.6042] [INSPIRE].

[25] Y. Hirono and M. Nitta, Anisotropic optical response of dense quark matter under rotation: compact stars as cosmic polarizers, Phys. Rev. Lett. 109 (2012) 062501 [arXiv:1203.5059] [INSPIRE].

[26] J.W.T. Hessels et al., A radio pulsar spinning at 716 Hz, Science 311 (2006) 1901 [astro-ph/0601337] [INSPIRE].

[27] K. Iida, T. Matsuura, M. Tachibana and T. Hatsuda, Melting pattern of diquark condensates in quark matter, Phys. Rev. Lett. 93 (2004) 132001 [hep-ph/0312363] [INSPIRE]. 
[28] H. Kawamura and S. Miyashita, Phase transition of the two-dimensional Heisenberg antiferromagnet on the triangular lattice, J. Phys. Soc. Jpn. 53 (1984) 4138.

[29] T. Okubo and H. Kawamura, Signature of a $Z_{2}$ vortex in the dynamical correlations of the triangular-lattice Heisenberg antiferromagnet, J. Phys. Soc. Jpn. 79 (2010) 084706 [arXiv: 1004.4730].

[30] E.J. Ferrer and V. de la Incera, Magnetic fields boosted by gluon vortices in color superconductivity, Phys. Rev. Lett. 97 (2006) 122301 [hep-ph/0604136] [InSPIRE].

[31] E.J. Ferrer and V. de la Incera, Chromomagnetic instability and induced magnetic field in neutral two-flavor color superconductivity, Phys. Rev. D 76 (2007) 114012 [arXiv: 0705.2403] [INSPIRE].

[32] W. Vinci, M. Cipriani and M. Nitta, Spontaneous magnetization through non-Abelian vortex formation in rotating dense quark matter, Phys. Rev. D 86 (2012) 085018 [arXiv: 1206.3535] [INSPIRE].

[33] M. Campostrini, P. Rossi and E. Vicari, Monte Carlo simulation of $C P^{N-1}$ models, Phys. Rev. D 46 (1992) 2647 [InSPIRE].

[34] M. Campostrini, P. Rossi and E. Vicari, Topological susceptibility and string tension in the lattice $C P^{N-1}$ models, Phys. Rev. D 46 (1992) 4643 [hep-lat/9207032] [INSPIRE]. 